\title{
Die Einwilligung von Minderjährigen in medizinische Eingriffe
}

Christian Peter

1 Entscheid des Bundesgerichts vom 2. April 2008 (2C_5/2008).

2 Gesetzliche Grundlagen: z.B. BE: Art. 40 Gesundheitsgesetz; ZH: § 20 Patientinnen- und Patientengesetz; BS: § 11 Spitalgesetz; AG: § 15 Patientendekret; GE: Art. 48 Loi sur la santé; VD Art. 23 Loi sur la santé publique.

Korrespondenz:

Dr. iur. Christian Peter

Rechtdienst Spitallietung

Inselspital

CH-3010 Bern

christian.peter@insel.ch

\section{Zusammenfassung}

Bei der Behandlung von Minderjährigen stellt sich oft die Frage, wer die Einwilligung in den Eingriff geben muss. Ist es die minderjährige Person selbst oder ein Elternteil? Das Bundesgericht hat sich vor kurzem mit dieser Frage auseinandergesetzt. Es hat einen Osteopathen zu einer Busse verurteilt, weil er den Willen eines 13-jährigen Mädchens nicht respektierte und gegen dessen Willen eine Behandlung durchführte [1].

Das Bundesgericht liess sich dabei vom Grundsatz leiten, dass vor jeder ärztlichen Behandlung die entsprechende Einwilligung des Patienten einzuholen ist [2]. Sei es, dass der urteilsfähige Patient selbst einwilligt, oder sei es, dass der gesetzliche Vertreter einwilligt. Lehnt ein Patient oder seine gesetzliche Vertretung eine Untersuchung, eine Behandlung oder einen Eingriff ab, darf grundsätzlich keine ärztliche Massnahme durchgeführt werden, ansonsten der Eingriff rechtswidrig wäre.

\section{Der Fall}

Ein 13-jähriges Mädchen musste sich wegen Schmerzen am Steissbein in Behandlung begeben. Die Ärztin schlug zwei Behandlungsmöglichkeiten vor: entweder nichts zu unternehmen oder das Steissbein durch einen Osteopathen zu repositionieren.

Dem Wunsch der Mutter und des Kindes folgend, wurde die Minderjährige an einen Ostheopathen überwiesen. Dieser nahm einen ersten Versuch vor, dass Steissbein zu repositionieren. Bereits während dieser ersten Behandlung schrie die Patientin ohne Unterbruch, verspannte sich und verweigerte die Kooperation. Die erhoffte Wirkung blieb aus, und der Osteopath unternahm trotz Flehen seiner Patientin, er solle die Behandlung nicht durchführen, einen zweiten Versuch. Die Mutter der minderjährigen Patientin verfolgte die Behandlungen taten- und wortlos.

Vier Tage später beschwerte sich die Mutter der Patientin bei der kantonalen Ärztegesellschaft. Der zuständige Gesundheitsdirektor leitete ein Verfahren gegen den betreffenden Ostheopathen ein, und dieser wurde wegen eines Verstosses gegen das kantonale Gesundheitsgesetz zu einer Busse von 1500 Franken verurteilt. Es wurde als erwiesen betrachtet, dass der Verurteilte ohne Einwilligung der urteilsfähigen

\section{Consentement des mineurs lors}

\section{d'actes médicaux}

Lors du traitement de mineurs, la question du consentement se pose fréquemment. Est-ce le mineur lui-même ou l'un de ses parents qui doit donner son accord? II y a peu, le Tribunal fédéral a légiféré en la matière en condamnant un ostéopathe à une amende pour ne pas avoir respecté la volonté d'une jeune fille de 13 ans et effectué le traitement contre son gré [1].

Dans sa décision, le Tribunal fédéral s'est laissé guider par le principe que tout traitement médical nécessite le consentement du patient [2]. Peu importe, qu'il s'agisse du consentement du patient capable de discernement ou de celui de son représentant légal. En effet, si un patient ou son représentant légal refuse un examen, un traitement ou une intervention, aucun acte médical ne doit en principe être pratiqué car cela le rendrait contraire à la loi.

Patientin eine Behandlung durchgeführt hatte. Dieser Entscheid wurde sowohl durch das Verwaltungsgericht als auch durch das Bundesgericht gestützt.

\section{Die Argumente vor Gericht und das Urteil des Bundesgerichts}

Der Beklagte argumentierte, die Mutter sei dabei gewesen und dadurch habe er angenommen, dass sie ihre Tochter vertrete. Durch ihr passives Verhalten habe sie ihr Einverständnis manifestiert. Zudem sei die Patientin ohnehin nicht urteilsfähig gewesen, weil sie geweint habe, als sie erfahren hatte, dass sie in den nächsten sechs Wochen nicht mehr werde reiten können. Aufgrund ihrer Sorge darum und ihrer Angst sei sie sowieso nicht in der Lage gewesen, die Situation richtig einzuschätzen und zwischen den beiden Behandlungsmethoden zu wählen.

Diese Argumentation vermochte das Bundesgericht nicht zu überzeugen. Es hielt fest, dass die 
Minderjährige nur dann durch die Mutter vertreten worden wäre, wenn ihre Urteilsfähigkeit zu verneinen gewesen wäre. Das Bundesgericht bejahte hingegen, dass das 13-jährige Mädchen im vorliegenden Fall fähig gewesen war, ihre Krankheit zu verstehen sowie die Auswirkungen der Entscheidung zu erkennen. Der Umstand, dass die Auswirkung des Entscheides gering warzur Erinnerung: die Unterlassung des Eingriffs war eine gleichwertige Behandlungsoption -, hatte u.a. zur Folge, dass bereits bei der 13-jährigen die Urteilsfähigkeit bejaht wurde.

Mit dem als Verteidigung gedachten Argument des Beklagten, er hätte sich anders verhalten, wenn das Mädchen alleine gekommen wäre, setzte er sich in Widerspruch zu seiner eigenen Argumentation und lieferte dem Gericht den Beweis, dass auch er grundsätzlich von der Urteilsfähigkeit der Patientin ausgegangen war, jedoch fälschlicherweise angenommen hatte, aufgrund der Begleitung durch die Mutter vertrete diese ihre Tochter.

Die Feststellung, dass die Mutter nicht frei von Schuld sei, änderte nichts an der Verurteilung, hatte jedoch Auswirkungen auf das Strafmass. Die Busse fiel - in Anbetracht des Bussenrahmens von 500 bis 200000 Franken - mit 1500 Franken relativ gering aus.

\section{Kommentar}

Wie eingangs erwähnt, verlangt das Bundesgericht, dass vor jeder ärztlichen Behandlung die Einwilligung des aufgeklärten Patienten einzuholen ist.

\section{Einwilligung}

Die Einwilligung kann mündlich, schriftlich oder auch konkludent (d.h. durch entsprechendes Verhalten) erklärt werden. Zu beachten ist, dass in einer allfälligen späteren Auseinandersetzung zwischen Arzt und Patient der Arzt die Beweislast für die Einwilligung trägt [3]. Daher ist die Einwilligung in den Behandlungsunterlagen zu dokumentieren.

Eine Einwilligung kann jedoch nur rechtsgültig erteilt werden, wenn die einwilligende Person aufgeklärt wurde und urteilsfähig ist.

\section{Aufklärung}

Die Aufklärung dient der Aufrechterhaltung der körperlichen Integrität und dem Schutz des Selbstbestimmungsrechts des Patienten [4]. Sie soll es dem Patienten ermöglichen, eine selbstverantwortliche Entscheidung zu treffen. Daraus folgt, dass der alleinige Adressat der Aufklärung der einwilligungsfähige Patient ist.

Der Arzt hat über den medizinischen Befund, die Behandlungsoptionen und deren möglichen Verlauf zu informieren. Die Aufklärung muss patientenspezifisch sein, soll die Grundlagen und die wesentlichen Elemente des Eingriffs erklären und dem Patienten eine Gewichtung der Risiken ermöglichen [5].

\section{Urteilsfähigkeit}

Der aufgeklärte Patient muss urteilsfähig sein, damit er rechtsgültig in eine Behandlung einwilligen kann. Auch Minderjährige können bezüglich einer Einwilligung in eine Behandlung urteilsfähig sein. Patienten in der Altersstufe von 12 bis 18 Jahren gelten als weitgehend urteilsfähig, soweit sie psychisch gesund und bei Bewusstsein sind. Die Urteilsfähigkeit wird aber fallbezogen beurteilt [6]. Im konkreten Fall muss geprüft werden, ob die jugendliche Patientin aufgrund ihrer geistigen Reife in der Lage ist, die Tragweite der Entscheidung richtig einzuschätzen, ihren Willen äussern und entsprechend handeln kann. Trifft dies zu, ist sie allein zuständig für die Erteilung der Einwilligung zu einem Eingriff.

$\mathrm{Zu}$ beachten ist, dass die Mündigkeit bzw. die Volljährigkeit bei dieser Frage keine Rolle spielt (siehe auch BGE 114 Ia 350 Erw. 7a). Die Urteilsfähigkeit orientiert sich nicht am Alter des Patienten, sondern an seinen geistigen Fähigkeiten.

Bestehen Zweifel an der Urteilsfähigkeit, können nachfolgende Kriterien helfen:

- Ist der Patient fähig, Informationen in Bezug auf die zu fällende Entscheidung zu verstehen und diese im Kontext eines kohärenten Wertsystems rational zu gewichten?

- Ist er fähig, die Situation und die Konsequenzen, die sich aus alternativen Möglichkeiten ergeben, richtig abzuwägen?

- Ist er fähig, den eigenen Willen zu äussern? [7]

Werden diese Fragen mit Ja beantwortet, kann von der Urteilsfähigkeit ausgegangen werden.

Der Patient, sei er nun mündig oder unmündig, hat in diesem Fall das Recht, selber seine Zustimmung zu ärztlichen Untersuchungen und Behandlungen zu geben [8]. Die Vertretungsbefugnis der Eltern entfällt also wie gesehen selbst bei minderjährigen Kindern, soweit sie in der Lage sind, die Situation bedarfsgerecht zu erfassen. Wichtig ist aber in diesem Zusammenhang, darauf hinzuweisen, dass alleine aus der Vernünftigkeit eines Entscheids über einen Eingriff nicht auf die Urteilsfähigkeit geschlossen werden darf.

Ist hingegen die Urteilsfähigkeit eines Patienten nicht gegeben, dann kann er nicht rechtsgültig in einen Eingriff einwilligen. Bei minderjährigen Patienten liegt in diesem Fall das Recht zur Einwilligung bezüglich des Eingriffs bei den Eltern (Art. 296 Abs. 1 ZGB i.V.m. 304 ZGB) [9], wobei jeder Elternteil das Recht alleine ausüben darf. 